\title{
An Efficient Method for Performance Monitoring of Active Phased Array Antennas
}

\author{
Benjamin Bräutigam, Marco Schwerdt, and Markus Bachmann
}

\begin{abstract}
Modern synthetic aperture radars (SARs) are equipped with active phased array antennas to electronically generate various antenna beams. The TerraSAR-X satellite is a high resolution SAR system launched in June 2007. Its active phased array X-band antenna hosts 384 transmit/receive modules (TRMs) for controlling the electronic beam steering in azimuth and elevation direction. The precise modeling of the antenna performance is only possible if the actual characteristics of each individual TRM are monitored. TerraSAR-X has been equipped with an innovative characterization mode based on a coding technique, which is the so-called pseudonoise gating method. The individual and simultaneous characterization of all TRMs is realized under most realistic conditions with power supply loads like in nominal radar operation. For the first time, this novel technique has been applied on a spaceborne SAR system.
\end{abstract}

Index Terms-Active phased array antenna, internal calibration, synthetic aperture radar (SAR), TerraSAR-X, transmit/ receive modules (TRMs).

\section{INTRODUCTION}

$\mathbf{T}$ HE FAST technological progress and success in remote sensing applications based on spaceborne synthetic aperture radars (SARs) lead to flexible radar systems to satisfy the user needs for global earth observation with diverse data products. Recent satellite SAR instruments are featuring various antenna beams and imaging modes. Many modern SAR systems operate an active phased array antenna to provide the multitude of different antenna beams within short switching times. The accurate monitoring of the antenna performance becomes necessary to achieve the high stability requirements of satellite SAR instruments.

The German satellite mission TerraSAR-X, launched in June 2007, has been designed as a flexible X-band SAR system implemented in a public-private-partnership between the German Aerospace Center (DLR) and EADS Astrium GmbH [1]. The satellite produces high-quality images in stripmap, spotlight, ScanSAR, and additional experimental modes for all polarizations in left and right looking mode. Covering a wide look angle range, in total, a variety of over 10000 antenna beams can be commanded in these different modes. The sensor operates at a center frequency of $9.65 \mathrm{GHz}$ with a maximum bandwidth of $300 \mathrm{MHz}$. Relevant TerraSAR-X system parameters are summarized in Table I.

Manuscript received May 7, 2008; revised October 1, 2008. Current version published March 27, 2009.

The authors are with the Microwaves and Radar Institute, German Aerospace Center, 82234 Wessling, Germany (e-mail: Benjamin.Braeutigam@dlr.de; marco.schwerdt@dlr.de; Markus.Bachmann@dlr.de).

Color versions of one or more of the figures in this paper are available online at http://ieeexplore.ieee.org.

Digital Object Identifier 10.1109/TGRS.2008.2008719
TABLE I

SYSTEM PARAMETERS OF TERRASAR-X

\begin{tabular}{lc}
\hline Centre Frequency & $9.65 \mathrm{GHz}$ \\
\hline Radiated RF Power & $2 \mathrm{~kW}$ \\
Bandwidth & $300 \mathrm{MHz}$ max \\
Antenna Array & 12 Panels by 32 Rows (384 TRMs) \\
Antenna Size & $4.8 \mathrm{~m}$ in azimuth, $0.7 \mathrm{~m}$ in elevation \\
Polarisation & $\mathrm{H}$ and V (single, dual, quad) \\
Look Angle Range & $15^{\circ}-60^{\circ}$ \\
Operation Modes & StripMap, ScanSAR, Spotlight \\
Antenna Beams & $>100$ elevation beams \\
& $>100$ azimuth beams \\
\hline Radiometric Stability & $<0.2 \mathrm{~dB}$ \\
Radiometric Accuracy & absolute $<0.6 \mathrm{~dB}$, relative $<0.3 \mathrm{~dB}$
\end{tabular}

TerraSAR-X products have an absolute radiometric accuracy of better than $0.6 \mathrm{~dB}$, inherently based on its relative radiometric accuracy and radiometric stability [2]. This high quality is guaranteed by calibrating the whole system during the commissioning phase after launch [3]. The accuracy of this calibration process essentially depends on the stability of the radar instrument and the capability to determine its radiometric characteristics. Instrument fluctuations and antenna pattern variations are the main error contributions to the radiometric stability. Thus, for monitoring and compensating drift effects down to individual RF components of its active front end, TerraSAR-X hosts an internal calibration facility [4].

For the various antenna beams, the active phased array antenna allows one to electronically steer and shape the patterns. The array consists of 384 slotted waveguide subarrays for horizontal and vertical polarizations arranged in a matrix of $N=12$ panels with $M=32$ rows. Each array element is individually adjusted in gain and phase by one active transmit/receive module (TRM) for shaping and steering of the antenna pattern in azimuth and elevation direction [5], [6].

Instrument stability is the prerequisite for successful calibration and high radiometric accuracy of a SAR system. The total instrument stability is determined by the internal calibration facility. In the case of drift or failure of individual TRMs, the antenna performance degrades. Only if the actual gain and phase settings - the beam excitation coefficients-are exactly known, the antenna beams can be accurately described.

In the module stepping mode of the ENVISAT Advanced SAR (ASAR) instrument [7], individual measurements on the excitation coefficients of the TRMs are only possible if all modules except the one being characterized are switched off. The power load conditions of this module stepping mode are nonrepresentative, as four ASAR TRMs are fed by one power supply. This leads to a less accurate gain and phase estimation 
compared to measurements in the nominal mode with all TRMs operating.

This paper shows the advantages of individual TRM characterization with the efficient pseudonoise (PN) gating method [8] implemented on TerraSAR-X [9]. After introducing the system features of TerraSAR-X, its active phased array antenna is presented in Section II. The concept of individual TRM characterization with details on TerraSAR-X specifics is described in Section III and is verified with on-ground tests in Section IV. For the first time, in-orbit characterization results with this coding technique are presented (Section V) in the frame of TerraSAR-X calibration measurements. The accuracy of its performance monitoring capabilities is treated in Section VI.

\section{TerraSAR-X Active Phased Array Antenna}

The stability of antenna performance is guaranteed by two novel calibration approaches both implemented in the TerraSAR-X system. The first key element of dynamic antenna characterization is a mathematical antenna model covering all passive and active components of the radar front end. The second important part is an internal calibration facility monitoring the instrument stability. In orbit, the absolute power level is calibrated via external targets like transponders or corner reflectors [10], [11]. Hence, for antenna performance monitoring, only relative characterization results are of interest.

\section{A. Antenna Model Theory}

Due to the high amount of different beams and modes, the antenna was precisely characterized on ground instead of costly and time consuming far-field measurements during the mission. An antenna model was established to simulate the patterns before launch validated by a dedicated near-field measurement campaign with a high measurement accuracy of $0.2 \mathrm{~dB}$. Thus, the in-orbit verification can be reduced to a small number of beams [12], [13]. The antenna model calculates the beam shapes, considering different input parameters like the following:

- geometry of the antenna;

- beam excitation coefficients of all 384 array modules;

- an antenna performance matrix;

- embedded radiation patterns measured on ground on single subarrays of the antenna.

The antenna geometry is well known from the spacecraft structure. The beam excitation coefficients are defined by the applied gain and phase settings of each array element to form the pattern.

The antenna performance matrix $\tilde{X}_{m n}$ contains information on drift and failures of individual array elements for calculating the antenna patterns. Derived from housekeeping telemetry data or individual module measurements, the actual performance is considered for adapting the individual excitation coefficients.

The embedded radiation patterns $G_{m n}(\epsilon, \alpha)$ are a superposition of single subarray patterns measured over azimuth angle $\alpha$ and elevation angle $\epsilon$. Each TRM excites one radiating subarray with a complex signal

$$
x_{m n}=a_{m n} \cdot \mathrm{e}^{j \varphi_{m n}}
$$

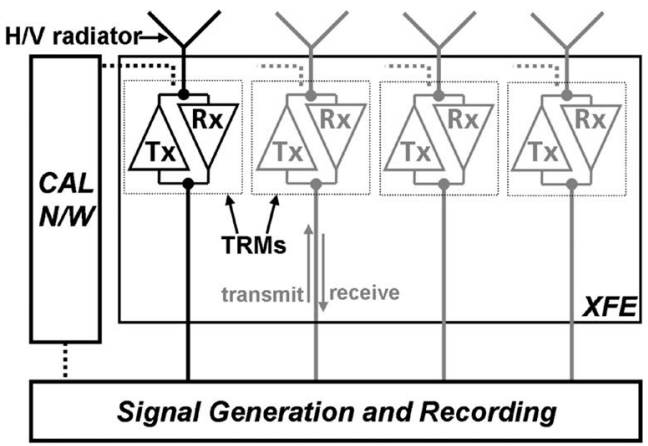

Fig. 1. XFE of TerraSAR-X instrument with four of 384 TRMs. The calibration signal is routed via couplers at the TRMs and the calibration facility network (CAL N/W).

where $m$ and $n$ are the indices of the $M$ rows and $N$ panels in the antenna, and the complex beam excitation coefficient $x_{m n}$ consists of amplitude $a_{m n}$ and phase $\varphi_{m n}$.

Combining the theory of array antennas [14] with the different inputs described earlier, the antenna generates the 2-D pattern $E(\epsilon, \alpha)$

$$
\begin{aligned}
E(\epsilon, \alpha)= & \sum_{m=0}^{M-1} \sum_{n=0}^{N-1} x_{m n} \cdot G_{m n}(\epsilon, \alpha) \cdot \mathrm{e}^{-j k \sin \alpha\left(n-\frac{N-1}{2}\right) \Delta x} \\
& \cdot \mathrm{e}^{-j k \sin \epsilon\left(m-\frac{M-1}{2}\right) \Delta y} .
\end{aligned}
$$

$\Delta x$ and $\Delta y$ are the subarray spacings in row and panel directions, and $k$ is the wavenumber.

\section{B. Instrument Internal Calibration Architecture}

For calibrating and monitoring the instrument stability, the radar instrument of TerraSAR-X features an internal calibration facility coupling into an additional port of each TRM, as shown in Fig. 1. Calibration pulses are routed through the X-band front end (XFE) to characterize critical elements of the transmit (TX) and receive (RX) paths. These pulses are directly looped back to the recording unit. The acquired signals can only be measured at the composite ports of the distribution networks located in the "signal generation and recording" unit. These signals can be evaluated for total instrument gain and phase. Periodical measurements monitor the instrument stability for possible gain and phase drifts. The TerraSAR-X in-orbit instrument stability is presented in [4].

\section{INDIVIDUAL TRM CHARACTERIZATION APPROACH}

Even though the TerraSAR-X XFE is designed to be insensitive to degradations like those of individually failed or drifting modules, it is necessary to detect such failures and continuously characterize the TRMs. The precise modeling of the antenna performance is only possible when the actual characteristics of each individual TRM are known.

\section{A. Estimation of Individual TRM Characteristics}

The tapering and steering of the antenna beam depend on the beam excitation coefficients $x_{m n}$ defining the gain and phase of the TRMs. Thus, apart from measuring the stability 


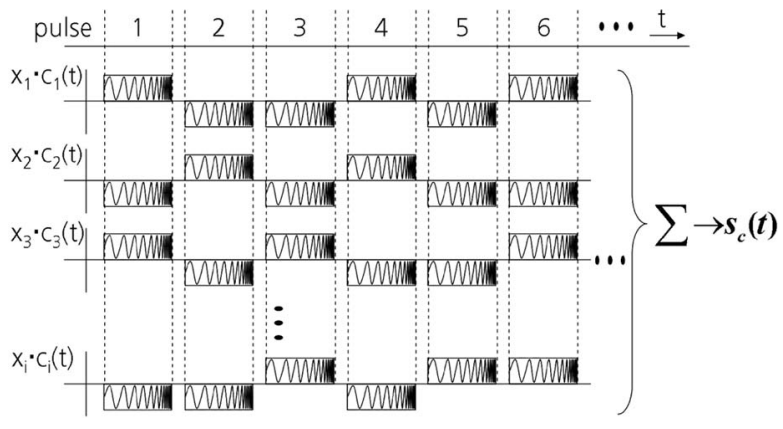

Fig. 2. Superposition of signals of all TRMs. Each signal is scrambled by its own code sequence applied from pulse to pulse.

of the instrument, it is necessary to retrieve information on the performance of individual TRMs. The actual status of each TRM setting has to be known, particularly considering performance degradation or malfunction. Comparing telemetry data (e.g., voltage and temperature behaviors of the TRMs) to appropriate on-ground characterization only provides limited information on the radar performance. The direct RF measurements of individual TRMs would only be possible if all modules except the one being characterized are switched off. This socalled module stepping procedure - as used for the ENVISAT ASAR instrument [7] - does not represent the actual status of operating modules due to different power supply loading in this mode.

A detailed analysis of individual TRMs within an active phased array antenna can be achieved by a coding technique, which is the so-called PN gating method, developed at the DLR [8]. The name "PN gating method" refers to the possibility of scrambling the TRM signals with a PN code. The advantage of this technique is that individual TRMs are characterized while all modules are operating, i.e., a characterization under most realistic conditions. In this special internal calibration mode, the actual phase of each TRM is shifted by $\pm 90^{\circ}$ from pulse to pulse according to a unique code sequence $c_{m n}$

$$
c_{m n}=\mathrm{e}^{ \pm j 90^{\circ}} .
$$

Changing the phase of a signal by a total of $180^{\circ}$ means to alternate its sign, depending on the code bit position but keeping its magnitude constant (see Fig. 2).

The total phase commanded for a TRM is the phase $\varphi_{m n}$ of its setting plus a shift by $\pm 90^{\circ}$. Consequently, the superposition of all TRM gains $a_{m n}$ and phases $\varphi_{m n}$ at the composite port of the distribution network yields the composite signal $s_{c}(t)$, as shown in Fig. 2

$$
s_{c}(t)=\sum_{m=0}^{M-1} \sum_{n=0}^{N-1} c_{m n}(t) \cdot a_{m n} \mathrm{e}^{j \varphi_{m n}}+n_{m n}
$$

where $a_{m n}$ and $\varphi_{m n}$ are assumed to be constant during its short measurement time of about $1 \mathrm{~s}, t$ defines the time position inside the code sequence, and $n_{m n}$ is the TRM inherent noise. Thus, the individual TRM signal is only changed over time by varying its sign $c_{m n}(t)$. To extract the information for one TRM, the composite signal is correlated with its conjugate complex code sequence. By this correlation process, the code modulation is removed, and the complex correlation peak represents an estimation of gain setting $\tilde{a}_{m n}$ and phase setting $\tilde{\varphi}_{m n}$ of the respective TRM

$$
\begin{aligned}
& \tilde{x}_{m n}=s_{c} \otimes c_{m n}^{*} \\
& \tilde{x}_{m n}=\int s_{c}(t) \cdot c_{m n}^{*}(t) \mathrm{d} t=\tilde{a}_{m n} \mathrm{e}^{j \tilde{\varphi}_{m n}} .
\end{aligned}
$$

All estimated excitation coefficients are summarized in the antenna performance matrix $\tilde{X}_{m n}$ of $M$ rows and $N$ columns (panels) according to the active antenna array of the SAR instrument

$$
\tilde{X}_{m n}=\left(\begin{array}{ccc}
\tilde{a}_{11} \mathrm{e}^{j \tilde{\varphi}_{11}} & \cdots & \tilde{a}_{1 N} \mathrm{e}^{j \tilde{\varphi}_{1 N}} \\
\vdots & \ddots & \vdots \\
\tilde{a}_{M 1} \mathrm{e}^{j \tilde{\varphi}_{M 1}} & \cdots & \tilde{a}_{M N} \mathrm{e}^{j \tilde{\varphi}_{M N}}
\end{array}\right) .
$$

This antenna performance matrix is fed into the antenna model for beam optimization and recalculation of the actual antenna pattern [see (2)].

\section{B. Applicable Codes}

Simulations have shown the impact of different code types on the quality of the correlation process [3]. The orthogonal Walsh code [15] has proven its robustness for the TerraSAR-X system, showing no code error due to cross correlation. In contrast, PN codes with finite suppression of the cross correlation have a higher estimation error of the characterized signal amplitude and phase. In addition, they are sensitive to different antenna phase distributions. Orthogonal Walsh codes derived from Hadamard matrices have the advantage of symmetric and recursive construction for a matrix dimension to the power of two [16]. To keep the measurement time and data volume low, the applied code length $l$ shall be as short as possible. The number of array elements restricts the minimum code length

$$
l_{\text {module }}=2^{w} \geq N \cdot M
$$

with an integer value $w$. An example of an $8 \times 8$ Hadamard matrix is printed in

$$
H_{8 \times 8}=\left(\begin{array}{cccccccc}
1 & 1 & 1 & 1 & 1 & 1 & 1 & 1 \\
1 & -1 & 1 & -1 & 1 & -1 & 1 & -1 \\
1 & 1 & -1 & -1 & 1 & 1 & -1 & -1 \\
1 & -1 & -1 & 1 & 1 & -1 & -1 & 1 \\
1 & 1 & 1 & 1 & -1 & -1 & -1 & -1 \\
1 & -1 & 1 & -1 & -1 & 1 & -1 & 1 \\
1 & 1 & -1 & -1 & -1 & -1 & 1 & 1 \\
1 & -1 & -1 & 1 & -1 & 1 & 1 & -1
\end{array}\right) .
$$

Each TRM is assigned to one row of the matrix, i.e., it has one code sequence of length $l$. A code sequence element is valid for the time of one pulse. According to the sign of the matrix value, the phase of a TRM is shifted by $\pm 90^{\circ}$. Each code sequence is orthogonal to all other code sequences.

\section{TRM Characterization Modes of TerraSAR-X}

The PN gating mode of TerraSAR-X can be executed as the following three basic performance checks while all 384 TRMs 
are simultaneously operated:

- module level with a minimum of 512 code bits for 384 TRMs;

- panel level with a minimum of 16 code bits for 12 panels;

- row level with a minimum of 32 code bits for 32 rows.

As the antenna beams are realized by applying row-wise excitation settings for elevation steering and panelwise excitation settings for azimuth steering, row and panel level checks are well suited for characterizing the beam excitations. For row level check, all modules of one antenna row are assigned to the same code sequence. Thus, the row level check provides an averaged estimation of the excitation setting for each row and, consequently, the 32 antenna beam settings in elevation. Panel level check means averaging over all subarray modules within one panel. The final result of all panels describes the antenna azimuth settings.

Although the minimum number of bits for each PN gating measurement is sufficient, a longer code length helps to improve correlation quality. Thus, for TerraSAR-X, row and panel level measurements are executed with 64 code bits each. The total measurement time is driven by the number of pulses per bit and the commanded pulse repetition frequency. It is on the order of $1 \mathrm{~s}$ for each mode.

\section{Advanced TRM Failure Check}

For health check of the front end, it is necessary to get a reliable feedback on failed TRMs, as this is a strong indication for instrument contingencies. During satellite operations, a fast but reliable failure diagnosis is necessary. This can be realized with an advanced TRM failure check method, as failed TRMs are detected by lower power levels of the respective row and panel at the affected array element position.

The total number of measured calibration pulses can be significantly reduced by only measuring the antenna array on row and panel levels consecutively. The total code length of both code sequences from panel and row level checks is

$$
\begin{aligned}
l_{\text {panel }}^{\prime} & =2^{u} \geq N \quad l_{\text {row }}^{\prime}=2^{v} \geq M \\
l_{\text {panel }}^{\prime}+l_{\text {row }}^{\prime} & =2^{u}+2^{v} \geq N+M
\end{aligned}
$$

with integer values $u$ and $v$. For $N$ and $M$ greater than two, it can be derived that the total number of code pulses is less than for the code length $l_{\text {module }}$ of a module level check

$$
\begin{aligned}
N+M & <N \cdot M \\
l_{\text {panel }}^{\prime}+l_{\text {row }}^{\prime} & <l_{\text {module }} .
\end{aligned}
$$

In the case of TRM failure, the total power level will decrease. The defective TRM can be identified by a lower power level at positions $n$ and $m$ in the respective panel and row.

Fig. 3 shows the code length required for an array of $M$ rows and $N$ panels (with $M=N$ ), comparing both methods. Increasing array size leads to a lower increase of required code length for the advanced TRM failure check. For TerraSAR-X, the row and panel level checks need at least 48 different coded calibration pulses in sum $\left(l_{\text {panel }}^{\prime}=16 ; l_{\text {row }}^{\prime}=32\right)$. In contrast to module level check with 512 code bits, the advanced TRM failure check is up to ten times faster than individual TRM characterization.

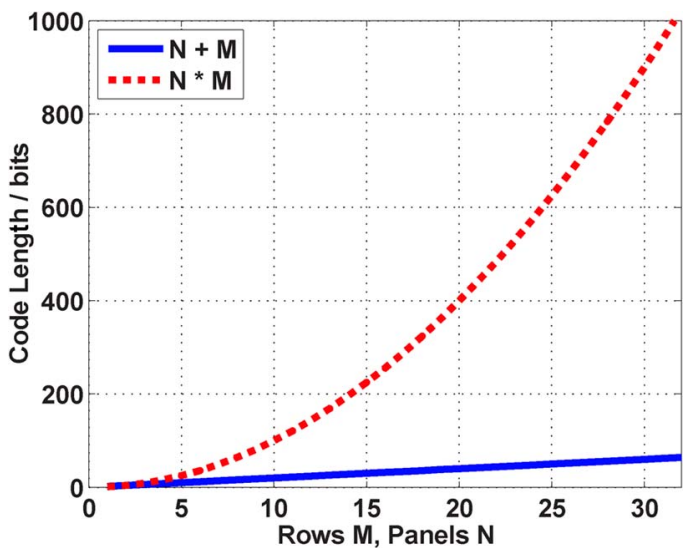

Fig. 3. Minimum code length for $M$ rows and $N$ panels with $M=N$.

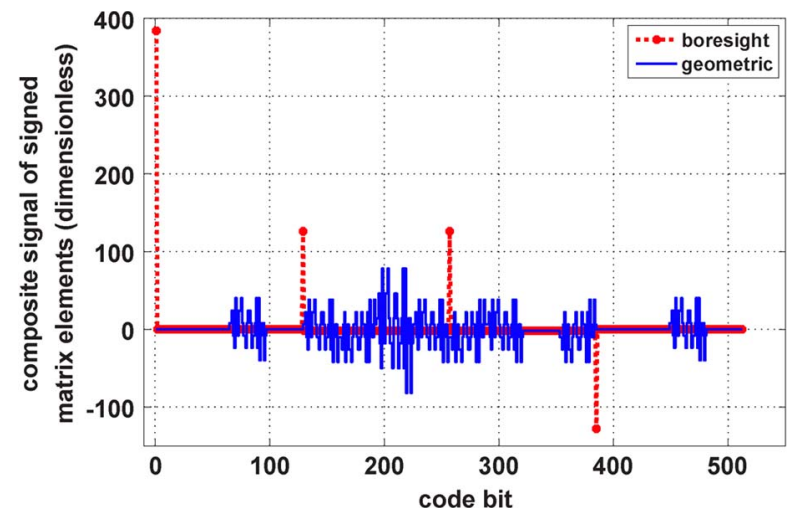

Fig. 4. Sum of 384 signals with signed code sequences of 512 code bits. The composite signal of boresight beam has a higher dynamic range than the geometric coding beam.

\section{E. Dynamic Range of Composite Signal}

For best signal-to-noise ratio in the internal calibration facility, the instrument is operated in boresight condition, i.e., all TRM gains and phases are set to equal and constant values during measurement. However, for PN gating with orthogonal Walsh codes, each TRM signal is furthermore modulated with a unique code (see Fig. 2).

Assuming equal gains for all TRMs, the composite signal reduces to a noiselike signal when the TRM phase excitations are scrambled. The only exception is for code bits where all signs are equal; compare the first column of (8). Here, the individual TRM signals coherently sum up to a high power level. Fig. 4 shows the sum of 384 coded signals over time in a module level check. The dashed line shows the high dynamic range for PN gating on boresight beam, which may lead to a nonideal saturation degree of the receiver.

Although TerraSAR-X is fully qualified for this high dynamic range of module level measurements, the drop of power level can be eliminated by choosing another underlying beam instead of boresight condition. While applying the PN gating code sequences to the TRMs over time, the underlying beam can have an additional phase distributed over the array antenna geometry. In an iterative process, the antenna phases are chosen by optimizing for the smallest dynamic range over time, when the overall sum signal is composed with the PN code sequence. 


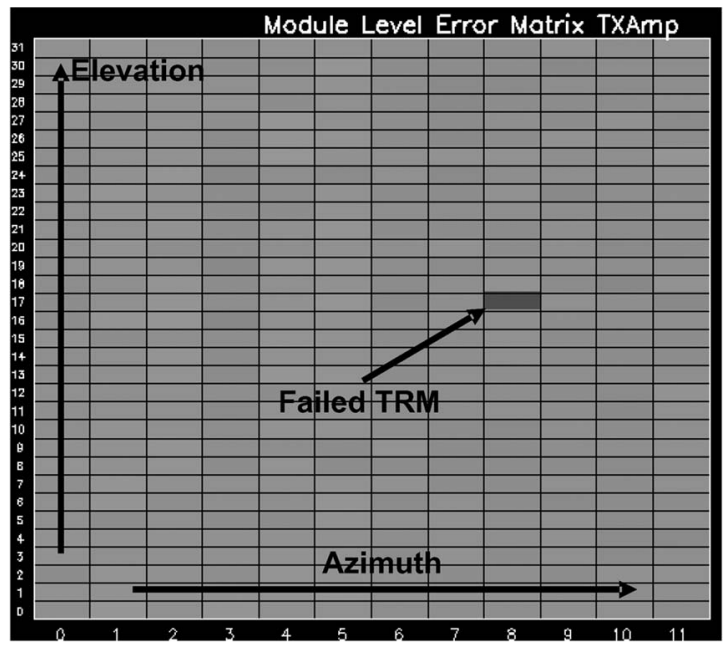

Fig. 5. Antenna performance matrix of TerraSAR-X antenna. Normalized TX amplitude estimation derived from module level check with one TRM switched off at position 8/17 (light gray: Full performance; dark gray: Failure).

By this "geometric coding," the underlying beam contributes an additional constant phase distribution during PN gating measurement, avoiding the coherent superposition of equally phased TRMs like in boresight condition. Thus, the power level of the composite signal for the first code bit is reduced, while the remaining part of the code sequence has more signal variation (see solid line in Fig. 4).

\section{ON-Ground Verification of Estimation Method}

The following several cases of performance degradation have been studied during on-ground verification activities:

1) single TRM failure;

2) malfunction of half a panel (16 TRMs) in a tapered beam;

3) gain and phase degradation of several TRMs.

The checks were executed as module, row, and panel levels on the TerraSAR-X antenna with 384 TRMs and compared to acquired reference sets without malfunction.

\section{A. TRM Failure From Module Level Check}

In the instrument configuration, one TRM was switched off. The results of a module level check are plotted into an antenna performance matrix representing the antenna TRM configuration of TerraSAR-X. Fig. 5 shows the estimated gains in TX. High power levels are light gray, and low power levels are dark gray. The evaluation shows a significant decrease in gain for the switched-off TRM. The position of the detected failed element is at the expected place that conforms to the assigned code at column 8 and row 17.

The degraded array element is written into the antenna performance matrix $\tilde{X}_{m n}$, which is an important input for the antenna model (6). Although few affected modules do not influence the total antenna performance, the antenna patterns can be recalculated in the case of several failures or module drifts.

\section{B. TRM Failure From Panel and Row Level Check}

In further measurement, the advanced TRM failure check from (12) is applied to the TerraSAR-X array with a switched-
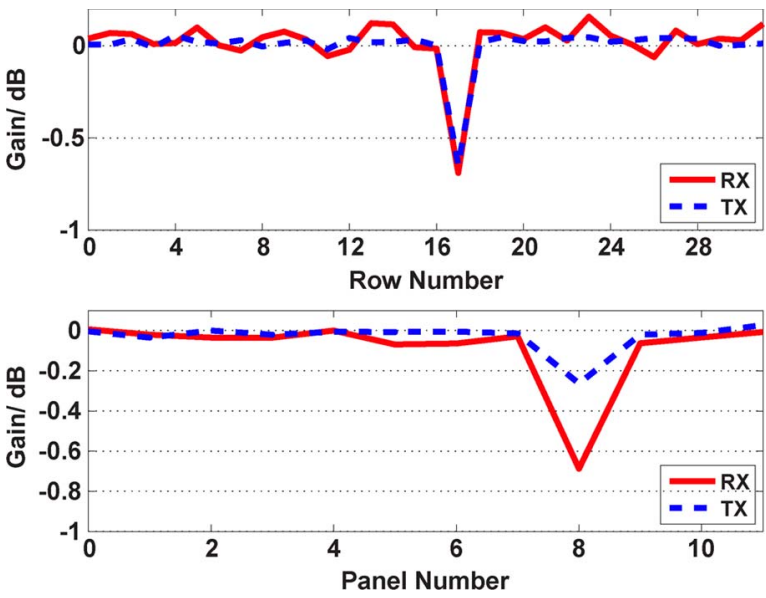

Fig. 6. (Top) Row level check of antenna with switched-off TRM. Gain degradation at row 17 is visible for TX and RX. (Bottom) Panel level check of antenna with switched-off TRM. Gain degradation at panel 8 is visible for TX and RX. Plots show the difference to reference measurement.

off TRM. Fig. 6 shows the effectiveness of this diagnosis method. It shows that the positions of failed modules are detected by reducing the analysis to panel and row level checks. As for module level check, the switched-off TRM can be identified by the lower gain in row 17 and panel 8 . The PN gating technique is accurate enough to show small differences on the order of few tenths of a decibel compared to nominal reference configuration.

Instead of applying a code of 512 bits length like for module level check, an equivalent meaningful result could be found much faster by combining two measurements of code length $l^{\prime}=64$ each for panel and row level checks. These two checks with shorter code lengths decrease the measurement time and data volume by a factor of four.

\section{Panel Malfunction in a Tapered Beam}

Applying the PN gating technique on a nominal tapered beam (like for stripmap mode) yields the actual beam coefficients set in the TRMs. The determined excitation can be compared to the commanded one and is fed into the antenna model for calculating the real antenna pattern.

Half a panel (16 TRMs) was switched off, whereas the antenna was excited with a gain taper in elevation direction. The commanded taper in elevation applied to all panels is drawn as a solid line in Fig. 7. By module level check, individual subarray elements are measured. Thus, the estimated gain over elevation can be analyzed for each panel separately. The measured elevation excitations are compared to the commanded ones. The estimated gain of panel 0 is plotted as a dashed line. It matches the commanded beam taper very well. Accordingly, the gain characteristics of (dotted line) panel 10 correspond to the expected values, too. The first 16 TRMs of panel 10 yield only noise signal, as these TRMs were switched off.

\section{Gain and Phase Estimation}

Several TRMs were commanded with different excitation coefficients in gain and phase compared to boresight operation. Panels 8 and 9 were commanded with a shift of $+45^{\circ}$ in TX 


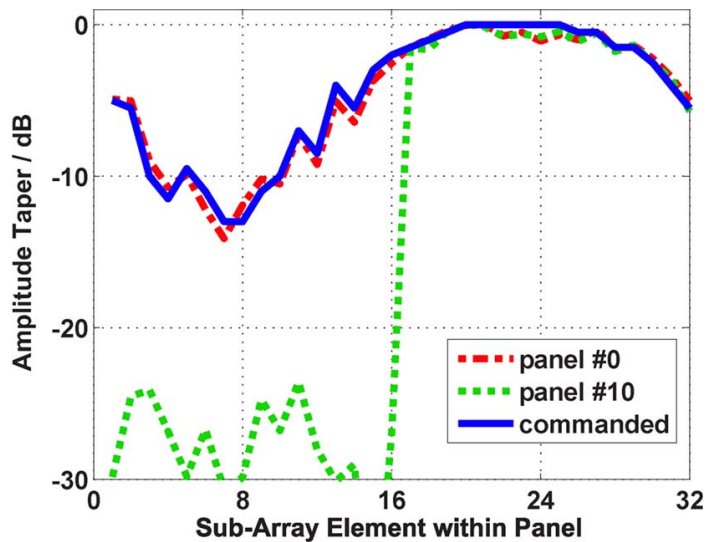

Fig. 7. Commanded and measured beam taper on panel 0 and panel 10 of TerraSAR-X antenna. The first 16 TRMs in panel 10 were switched off.
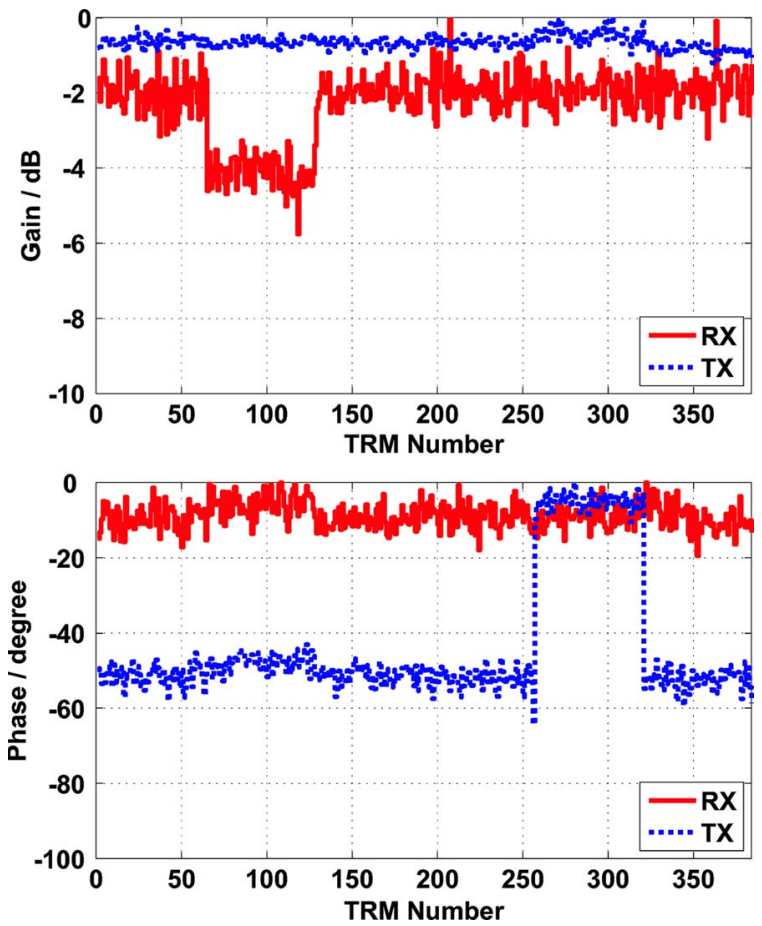

Fig. 8. On-ground measurements of antenna in TX and RX. Each data set is referenced to its maximum value. (Above) Gain degradation of $-2.4 \mathrm{~dB}$ in RX for TRMs 64 to 127 corresponding to panels 2 and 3. (Below) Phase degradation of $+45^{\circ}$ at TRMs 256 to 319 corresponding to panels 8 and 9 .

phase, while panels 2 and 3 were attenuated by $2.3 \mathrm{~dB}$ for RX gain. All other panels had nominal settings of $0 \mathrm{~dB}$ attenuation and $0^{\circ}$ phase in TX and RX.

Fig. 8 shows all TRM characteristics for TX and RX behaviors of the antenna. After averaging over all elements of one panel, the estimated TX phase of panels 8 and 9 was $+45^{\circ}$, as configured. The estimated RX gain of panels 2 and 3 results in $-2.4 \mathrm{~dB}$, only deviating by $0.1 \mathrm{~dB}$ from the commanded value.

After achieving these excellent results of the on-ground verification, PN gating is applied as an essential TRM characterization method for in-orbit performance monitoring. The total accuracy of repeated spaceborne measurements is summarized in the following sections.

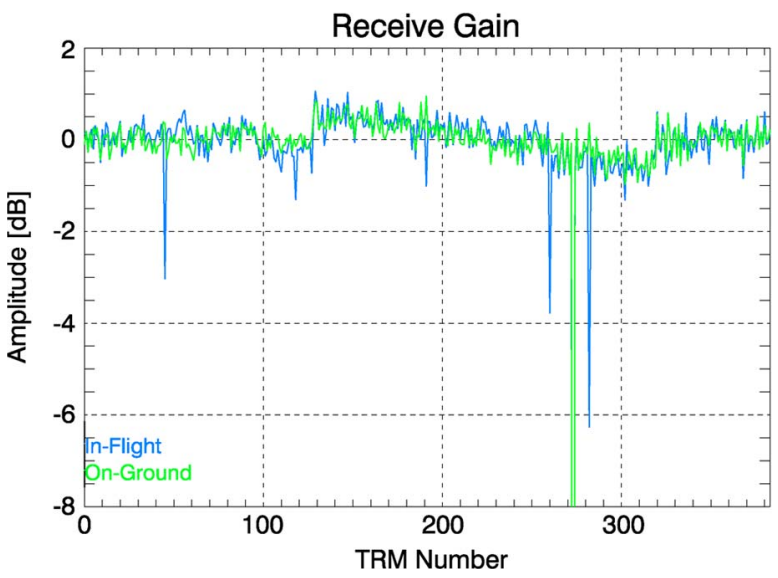

Fig. 9. Comparison of first in-flight measurement to on-ground reference. The RX gain of all TRMs is shown. On-ground and in-flight results are normalized to each other. TRM at position 273 is switched off and has a gain of less than $-20 \mathrm{~dB}$. TRMs at positions 45,260 , and 282 show degradations of more than $2 \mathrm{~dB}$ compared to their on-ground statuses.

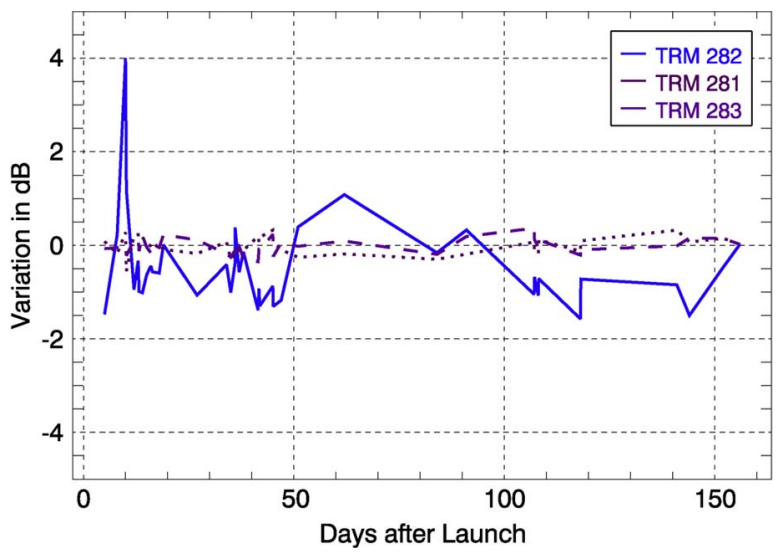

Fig. 10. Measurement variation of three neighbored TRMs over mission time. TRM 282 is one of the three degraded TRMs for the RX case. It has the highest variation of RX gain. Neighbored TRMs 281 and 283 show much more stable behavior.

\section{IN-Orbit TRM PERFormance MONITORING}

One of the first test data takes after satellite launch, and instrument switch-on was a PN gating TRM measurement. Aside from the functional verification of the mode, this gave a fast telemetry-independent look on the performance of the TRMs. There are 384 excitations each for TX and RX gains, as well as for TX and RX phases; thus, a total of 1536 estimations exist. The first in-flight results show very good accordance to the onground reference, as plotted for the RX amplitude in Fig. 9.

Comparing all 1536 measurements to their on-ground reference, more than $99 \%$ of all excitations correspond to the expected result. This proves the robustness of the PN gating technique, confirming the on-ground verification of this method. It has to be mentioned that one TRM was intentionally switched off before launch as it showed anomalies. This condition will be kept during the whole satellite mission to avoid negative influences (compare row 17 of panel 8 in Fig. 5). Three modules at position 45,260 , and 282 work outside the expected performance range in RX operation. Their actual degradation between 2 and $6 \mathrm{~dB}$ is successfully determined with repeated 

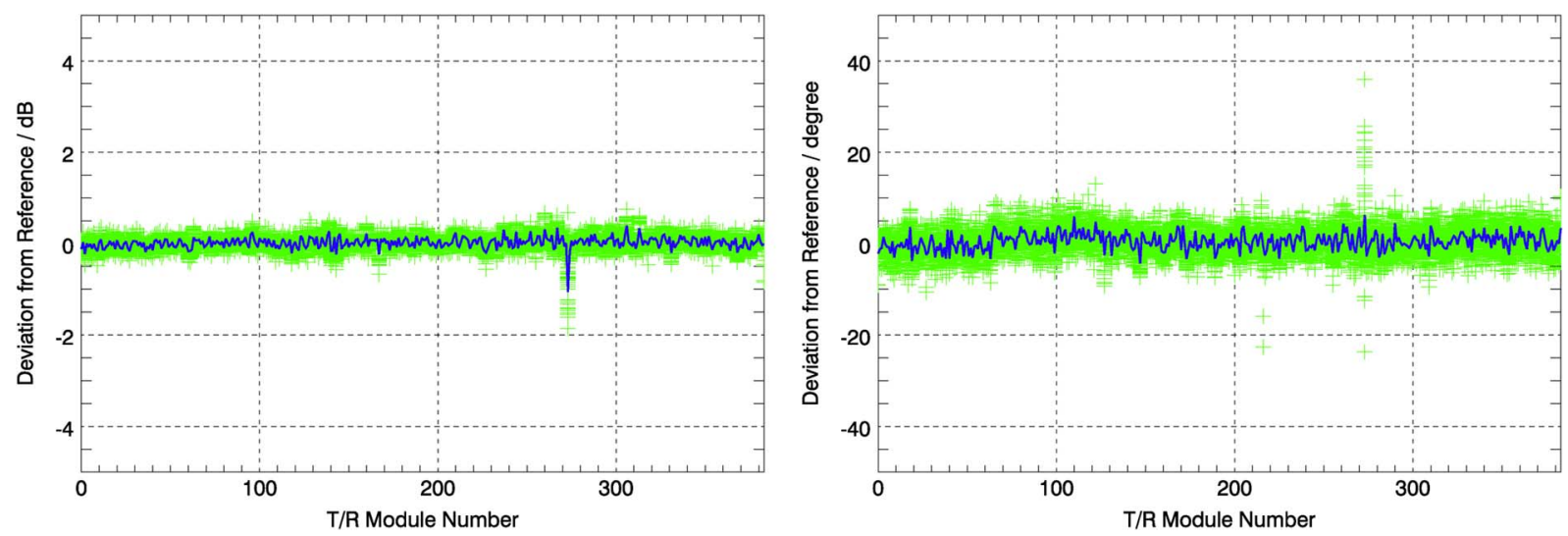

Fig. 11. In-orbit variation of individual TRMs for 42 different measurements of TX behavior. Each asterisk represents one evaluated measurement point. The solid curve represents the mean value of all measurement results per TRM. (Left) TX amplitude. (Right) TX phase.

TRM measurements during the commissioning phase. TRM position 282 has been identified with the highest variation over repeated measurements. In Fig. 10, the solid line shows its variation over time by plotting its derived excitation. The dashed lines are the neighbored TRMs plotted for comparison. As the long-term variation is still small enough for all TRMs, no action is necessary. However, the plots emphasize the need for long-term TRM performance monitoring and trend analysis. Considering the three degraded RX modules after launch, PN gating measurements become stringent in terms of efficient and robust individual TRM characterization.

\section{Measurement AcCuracy}

During the five months of commissioning phase, numerous PN gating checks have been executed in order to monitor the TRM performance. In total, the in-flight monitoring comprises the evaluation of about 100 data sets including the following:

- 42 module level acquisitions;

- 25 row level acquisitions;

- 25 panel level acquisitions.

The actual excitation of each module can be measured for TX and RX gains, as well as for TX and RX phases. Thus, in total, four times 384 measurements are available for each PN gating acquisition. For monitoring the stability, all evaluations are normalized to the first in-flight measurement. The individual TRM stabilities during the mission time are then easily visible.

Fig. 11 shows the estimated TX excitation settings derived from 42 module level measurements during the commissioning phase. The solid line through all points connects the mean values of these measurements to provide the average offset compared to the reference values. The evaluation of all settings shows a very stable behavior of the TRMs. The results provide the following two points of information for each element.

1) The reference offset of one element specifies the actual excitation coefficient (compared to boresight).

2) The standard deviation of each element comprises the setting accuracy of the TRMs, their variation, and the PN gating estimation accuracy.

Table II summarizes the standard deviation for the module, row, and panel level measurements. The TRM at position 273, which is switched off, has been masked out for this analysis, as noise signals falsify the measurement accuracy with their
TABLE II

StANDARD DEVIATION OF REPEATEd MEASUREMENTS

\begin{tabular}{llll}
\hline $1 \sigma$-Standard Deviation & Module Level & Row Level & Panel Level \\
\hline TX Amplitude & $0.092 \mathrm{~dB}$ & $0.023 \mathrm{~dB}$ & $0.025 \mathrm{~dB}$ \\
RX Amplitude & $0.188 \mathrm{~dB}$ & $0.062 \mathrm{~dB}$ & $0.183 \mathrm{~dB}$ \\
TX Phase & $1.91^{\circ}$ & $0.42^{\circ}$ & $1.23^{\circ}$ \\
RX Phase & $1.29^{\circ}$ & $0.32^{\circ}$ & $0.66^{\circ}$ \\
\hline
\end{tabular}

inherent gain and phase instabilities (see variations at position 273 in Fig. 11). Looking at the amplitude accuracies in Table II, the TRMs are more stable in TX. This is due to better signalto-noise ratio of the whole chain for TX than for RX. As the accuracy of the PN gating method itself is part of the overall measurement accuracy of the TX case, this new TRM characterization method is hence better than the following:

- $0.09 \mathrm{~dB}$ for amplitude;

- $1.9^{\circ}$ for phase.

Finally, the presented PN gating method demonstrates the high stability of the TerraSAR-X TRMs over the complete duration of the commissioning phase. The TRMs perform better than $0.1 \mathrm{~dB}$ for TX gain and $0.2 \mathrm{~dB}$ for RX gain, and $2.0^{\circ}$ for TX phase and $1.3^{\circ}$ for RX phase.

\section{CONCLUSION}

SARs with active phased array antennas depend on the reliable performance of its electronic instrument components. For the TerraSAR-X satellite, the stability of its 384 TRMs is of crucial importance for fast and flexible generation of various antenna beams. The flexibility of TerraSAR-X can be underlined with its ability to demonstrate new SAR modes and the introduction of innovative calibration concepts [2], [3].

Implementing the novel PN gating method is an important milestone for the successful calibration and dynamic optimization of the whole system during mission lifetime. The PN gating method allows operating all TRMs under most realistic conditions with the advantage that all individual modules can be characterized simultaneously. During on-ground characterization of the TerraSAR-X radar instrument, this technique has already been established as an essential diagnostic tool for functional checks, as well as TRM drift and failure monitoring. 
PN gating acquisitions are possible as different level checks based on module, panel, and row-wise measurements. Module level checks provide precise estimations of TRM characteristics in amplitude and phase, detecting performance degradation or module failure of individual TRMs. Combined row and panel level checks accelerate the process of TRM failure detection by localizing gain degradation inside the whole antenna.

For the first time ever, this innovative method is applied in a spaceborne environment on the TerraSAR-X mission. The results from repeated in-orbit measurements prove the high estimation accuracy of the PN gating technique of better than $0.09 \mathrm{~dB}$ for amplitude and $1.9^{\circ}$ for phase estimation of individual TRMs.

The fast and accurate estimation of individual TRM settings during the lifetime of the SAR instrument is a valuable support for in-orbit characterization and performance stability monitoring. As proven for TerraSAR-X, the evaluation of all measurements shows that PN gating is an excellent method for detecting TRM failures and characterizing instrument degradation. Furthermore, the technique described in this paper is applicable for the characterization and calibration of other advanced sensor systems featuring active phased array antennas.

\section{REFERENCES}

[1] S. Buckreuss, W. Balzer, P. Mühlbauer, R. Werninghaus, and W. Pitz, "The TerraSAR-X satellite project," in Proc. 25th IEEE Int. Geosci. Remote Sens. Symp., Toulouse, Frankreich, 2003, pp. 3096-3098.

[2] M. Schwerdt, B. Bräutigam, M. Bachmann, B. Döring, D. Schrank, and J. Hueso, "Final results of the efficient TerraSAR-X calibration method," in Proc. IEEE Radar Conf., Rome, Italy, 2008, pp. 1175-1180.

[3] M. Schwerdt, D. Hounam, B. Bräutigam, and J. L. Alvarez-Pérez, "TerraSAR-X: Calibration concept of a multiple mode high resolution SAR," in Proc. 25th IEEE Int. Geosci. Remote Sens. Symp., Seoul, Korea, 2005, pp. 4874-4877.

[4] B. Bräutigam, M. Schwerdt, and M. Bachmann, "In-flight monitoring of TerraSAR-X radar instrument stability," in Proc. IRS, Cologne, Germany, 2007.

[5] W. Pitz, "The TerraSAR-X satellite," in Proc. 6th Eur. Conf. Synthetic Aperture Radar, Dresden, Germany, 2006.

[6] M. Wahl, "High precision T/R module for SAR earth observation," in Proc. 6th Eur. Conf. Synthetic Aperture Radar, Dresden, Germany, 2006.

[7] C. Buck, "ASAR instrument gain monitoring," ESTEC, European Space Agency (ESA), ENVISAT ASAR Calibration Review (ECR), 2002.

[8] D. Hounam, M. Schwerdt, and M. Zink, "Active antenna module characterisation by pseudo-noise gating," in Proc. 25th ESA Antenna Workshop Satellite Antenna Technol., Noordwijk, The Netherlands, 2002.

[9] B. Bräutigam, M. Schwerdt, M. Bachmann, and M. Stangl, "Individual T/R module characterisation of the TerraSAR-X active phased array antenna by calibration pulse sequences with orthogonal codes," in Proc. 27th IEEE Int. Geosci. Remote Sens. Symp., Barcelona, Spain, 2007, pp. 5202-5205.

[10] B. Bräutigam, M. Schwerdt, and M. Bachmann, "The external calibration of TerraSAR-X, a multiple mode SAR-system," in Proc. 6th Eur. Conf. Synthetic Aperture Radar, Dresden, Germany, 2006.

[11] B. Döring, M. Schwerdt, and R. Bauer, "TerraSAR-X calibration ground equipment," in Proc. WFMN, Chemnitz, Germany, 2007, pp. 86-90.

[12] M. Bachmann, M. Schwerdt, B. Bräutigam, B. Grafmüller, A. Herschlein, and J. L. Alvarez-Pérez, "The TerraSAR-X antenna model approach," in Proc. ITG Conf. Antennas, Munich, Germany, 2007, pp. 139-142.
[13] M. Bachmann, M. Schwerdt, and B. Bräutigam, "TerraSAR-X in-orbit antenna model verification results," in Proc. EuRAD, Munich, Germany, 2007, pp. 9-12.

[14] in Antenna Theory: Analysis and Design. Hoboken, NJ: Wiley, 1997.

[15] S. D. Silverstein, "Application of orthogonal codes to the calibration of active phased array antennas for communication satellites," IEEE Trans. Signal Process., vol. 45, no. 1, pp. 206-218, Jan. 1997.

[16] J. H. Dinitz, Contemporary Design Theory: A Collection of Surveys. Hoboken, NJ: Wiley, 1992.

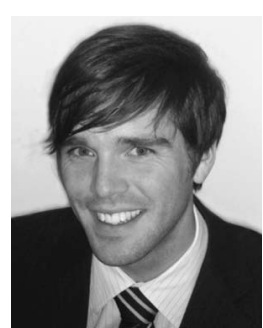

Benjamin Bräutigam received the Dipl.Ing. degree in electrical engineering from the Technical University Karlsruhe, Karlsruhe, Germany, in 2003.

In 2003, he was a Visiting Scientist at the Environmental Technology Laboratory of the National Oceanic and Atmospheric Administration, Boulder, CO. Since 2004, he has been with the Microwaves and Radar Institute, German Aerospace Center, Wessling, Germany. Since then, he has been in the radar calibration group responsible for internal instrument calibration in spaceborne SAR projects like TerraSAR-X, TerraSAR-L, TanDEM-X, and Global Monitoring Environment and Security Program Sentinel-1. His major research interests include the development and analysis of innovative methods for instrument calibration and performance monitoring.

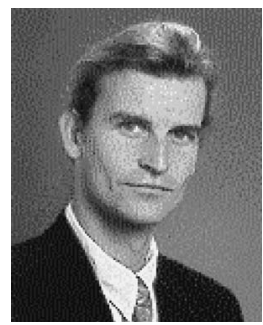

Marco Schwerdt received the Dipl.Ing degree in electrical engineering and the Doctorate (Dr. Ing.) degree, with a thesis on electrooptical E-field sensors, from the Technical University of Berlin, Berlin, Germany.

Since 1998, he has been with the Microwaves and Radar Institute, German Aerospace Center (DLR), Wessling, Germany, working on SAR calibration methods and performance analysis tools. Since 2000 he has been the head of the Radar Calibration Group, performing various radar calibration activities for different SAR missions like XSAR/SRTM or the ScanSAR mode of ASAR/ENVISAT. He is responsible for the successful calibration of the whole SAR system of the German TerraSAR-X satellite mission, launched in 2007. Currently, all preparations are made to calibrate the TanDEM-X system. Furthermore, as part of the Global Monitoring Environment and Security Program, he is responsible for developing the overall SAR system calibration and validation plan for the Sentinel-1 mission of the European Space Agency. Under his leadership, the DLR's comprehensive radar calibration facilities, including novel tools for product quality control and performance analysis, have been maintained and extended. His major research interest includes the development of innovative and efficient calibration methods.

Dr. Schwerdt received the Award "Der Deutsche Gründerfonds" in 1997 for establishing an enterprise of manufacturing electrooptical field sensors under the patronage of the German Federal Minister for Science and Research.

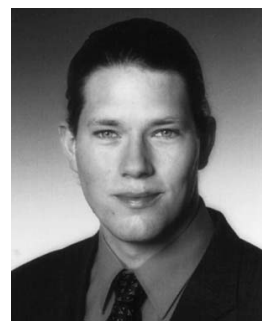

Markus Bachmann received the Dipl.Ing. degree in electrical engineering from the Technical University of Karlsruhe, Karlsruhe, Germany, in 2005.

Since 2005, he has been with the Microwaves and Radar Institute, German Aerospace Center, Wessling, Germany, where he is working as a Calibration Engineer in SAR calibration. In the TerraSAR-X project, he is responsible for the antenna model calibration, and in TanDEM-X for the digital elevation model calibration concept. 